\title{
Energy Efficient PNC Selection Procedure for the IEEE 802.15.3-Based HR-WPAN*
}

\author{
EunChang Choi ${ }^{1}$, JaeDoo Huh ${ }^{1}$, Soo-Joong Kim ${ }^{2}$, and WoongChul Choi ${ }^{3, * *}$ \\ ${ }^{1}$ Sensor Networking Research Team \\ Electronics and Telecommunication Research Institute \\ \{ecchoi, jdhuh\}@etri.re.kr \\ ${ }^{2}$ School of Electronic and Electrical Engineering, Kyungpook National University \\ sjkim@knu.ac.kr \\ ${ }^{3}$ Department of Computer Science \\ wchoi@daisy.kw.ac.kr \\ KwangWoon University, \\ Seoul, Korea 139-701
}

\begin{abstract}
In the IEEE 802.15.3-based HR-WPAN(High Rate Wireless Personal Area Networks), the selection of PNC is based on the capability of each DEV in the piconet. The selected DEV playing the role of a PNC will consume the most amount of energy among the DEVs in the piconet. Since the energy consumption of the PNC is closely related to its frame transmission range in the piconet, it is important to consider the relative location of each DEV when selecting the PNC among DEVs. In this paper, we propose a novel approach to the PNC selection procedure in the piconet that attempts to position the PNC at the center of the topology. By doing so, it can minimize the total transmission distances from the PNC to all other DEVs, resulting in saving energy for the PNC and therefore extending the life-time of the PNC as well as the piconet. We describe our method in detail and present experimental results that show the significant improvement in the energy saving.
\end{abstract}

Keywords: IEEE 802.15.3, HR-WPAN, Superframe, Beacon, Piconet, PNC (Piconet Cooordinator).

\section{Introduction}

HR-WPAN(High Rate Wireless Personal Area Networks) is the network technique which provides the communication service for the short distance, around 10 meters indoor or closed public place. HR-WPAN connects PCs, cellular phones, electronic appliances, mobile devices and other machines wirelessly, and provides inter-device communication for supporting variety applications[1][2]. In HR-WPAN, the desired $\mathrm{DEV}($ Device) are gathered tighter, and they make Piconet for communicating each

\footnotetext{
* This research has been conducted by Korea Science and Engineering Foundation under contract number R01-2005-0000-10934-0 and by the Research Grant of KwangWoon University in 2006.

** Corresponding author.
} 
other. The most capable DEV in Piconet becomes PNC(Piconet coordinator) and manages communication among DEVs. Generally, when they find DEV which has the capability to manage piconet, they choose it as PNC. PNC performs the most frequent communications during managing piconet, so PNC consumes much more energy than other DEVs. The power saving issue of the battery-using DEV in wireless network is researched actively. The power saving of DEV in wireless networks is an important issue, too[3][4][5]. In this paper, we propose the procedure to save power at the most power consumer, PNC.

The rest of this paper is organized as follows. In Section 2, we review the PNC handover of the IEEE 802.15.3 standard. We provide the problem statements in Section 3 and detailed description of PNC handover and its performance analysis in Section 4. We conclude the paper in Section 5.

Table 1. Comparison order of fields for PNC handover

\begin{tabular}{|c|c|c|}
\hline Order & Information & Note \\
\hline 1 & $\begin{array}{c}\text { PNC Des-mode bit in capabilities } \\
\text { field }\end{array}$ & PNC Des-mode=1 is preferred \\
\hline 2 & SEC bit in PNC capabilities field & SEC $=1$ si preferred \\
\hline 3 & PSRC bit in PNC capabilities field & PSRC $=1$ is preferred \\
\hline 4 & Max associated DEVs & Higher value is preferred \\
\hline 5 & Max CTRqBs & Higher value is preferred \\
\hline 6 & Transmitter power level(PHY \\
dependent) & Higher value is preferred \\
\hline 7 & $\begin{array}{c}\text { MAX PHY rate (PHY dependent) } \\
\text { DEV address }\end{array}$ & Higher value is preferred \\
\hline 8 & \multicolumn{2}{c}{ Higher value is preferred } \\
\hline
\end{tabular}

\section{IEEE 802.15.3 PNC Handover}

For the communication between DEVs in IEEE 802.15.3, it starts when the DEV which wants frame transmission makes piconet. In IEEE 802.15.3, the piconet is the wireless ad-hoc data communication system which makes that many DEVs can communicate each others. In the piconet, DEV can communicate to the all the directions inside of 10 meters. DEV which initiate the piconet becomes PNC, and manages communication of DEVs in the piconet. PNC transmits the piconet information to put in the beacon.. After the piconet is initialized, PNC can hand over the PNC role to the most capable DEV.

PNC handover happens when PNC DEV leaves the piconet, or delegates the role to other DEV. Before the role handover, PNC should choose the proper DEV which 
have the capability to do PNC role. Many DEVs in the piconet can become PNC, but the most capable DEV will be the PNC after selection process.

Table 1 shows the process of selecting PNC. As shown in Table 1, PNC hands over its role after the comparison, if another DEV has the better capability[1]. In IEEE 802.15.3 standard, they consider only DEV's own capability during selecting PNC, but the power consumption in the PNC role is not considered. They just consider whether DEV uses the outer power or battery by using the PSRC bit.

\section{Problem Statement}

In the IEEE 802.15.3 standard, the PNC selection procedure considers only the capability of each DEV. It does not consider the anticipated power that would be consumed by the DEV playing the role of PNC. That is, a DEV with the best capability will play the role of PNC no matter where it is located in the piconet.
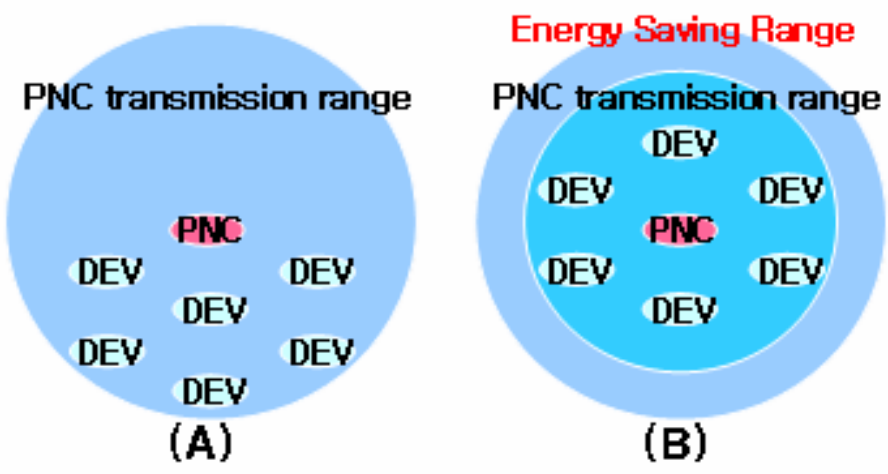

Fig. 1. Energy consumption range based on the location in the piconet

As shown in Fig. 1, the PNC in A has a broader minimum transmission range required to communicate with all DEV's than that of the PNC in B assuming the same topology. Even though the PNC in A might have a better capability as a DEV than that of the PNC in B, it will consume more energy when it plays the role of a PNC. As a result, in situation $\mathrm{A}$, the amount of time for which the selected DEV can assume the role of a PNC will be reduced and unnecessary PNC handover could occur. Thus, if one can select a DEV that can consume the minimum amount of power for transmission by considering the relative location of each DEV in the piconet topology, the life-time of a PNC will be extended.

This paper proposes a novel approach to the PNC selection procedure that chooses the center DEV by taking into consideration the relative location of each DEV in the piconet topology as well as their capabilities. This will lead to extending the life-time of a PNC by saving the power that could be consumed due to the unnecessary PNC handovers. 


\section{Energy Efficient PNC Selection Procedure}

In order to save energy, we want to keep the power needed for transmission at the minimum possible level while still allowing other DEVs in the piconet to receive signals properly. To do that, it is important to position the DEV that will play the role of a PNC at the center of a piconet topology. Moreover, even if we can find a DEV at the center of the topology, we should also consider whether that the DEV can perform the function as the PNC of the piconet. Thus, in this paper, we propose an energyefficient PNC selection procedure that checks the location of each DEV in addition to the existing PNC handover procedure.

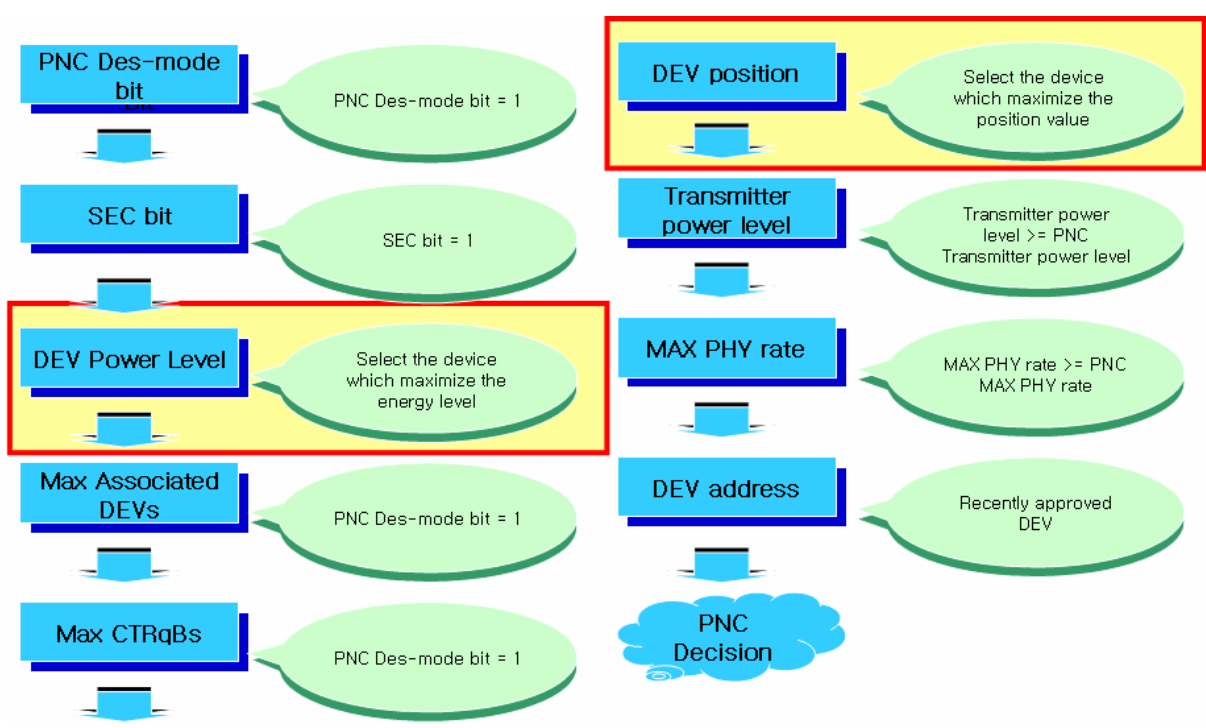

Fig. 2. PNC handover process considering the location and the remaining battery power of DEVs

Fig 2 shows the outline of our proposing PNC selection procedure in the existing PNC handover process. The boxed area describes the process for the PNC positioning. The PNC decides upon whether to handover by comparing its capability with other DEV through the process shown in Fig 2. Even if a DEV is positioned at the center of the topology, it can not become the PNC in case it does not satisfy the necessary conditions for the PNC. In the last phase of the selection process in Fig 2, it will select the DEV with the most remaining battery power if none of the DEVs use external power. Since our approach takes the location of the PNC for saving energy into accunt, it makes sense to select the PNC with the most remaining battery power among the DEVs with the same condition.

\subsection{PNC Selection Algorithm with Energy Level Consideration}

A PNC handover process will be invoked when the battery level of the current PNC goes below a threshold value. Therefore, the energy level of a node should be consid- 
ered before the topological location of the node. Power connectivity is checked only for a node in the current IEEE 802.15.3 standard. In this paper the energy level of a node will be also considered for a PNC selection.

\subsection{Finding Location of Each DEV for PNC Selection}

To find the relative location of each DEV in the topology, we propose using the transmission signal power from each DEV. It is possible for each DEV in the piconet to measure the signal power from other DEV. Each DEV can estimate their relative locations in the topology by taking into account all the signal powers from other DEVs.

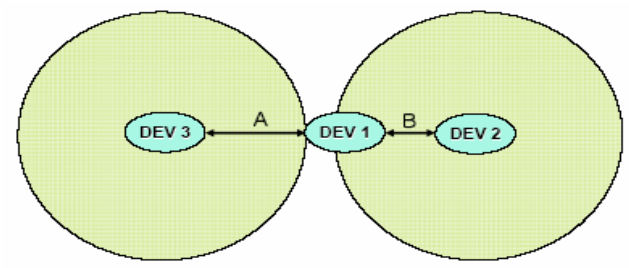

Fig. 3. The relative strengths of signals based on distances between DEVs

Fig 3 shows the situation where DEV1 receives the signals from both DEV2 and DEV3 and DEV2 is closer to DEV1 than DEV3 is. In this situation, assuming that both DEVs transmitting to DEV1 with the same signal strength, DEV1 will receive a stronger signal from DEV2 than that from DEV3 because the signal strength is inversely related to their relative distances [6]. Since each DEV receives stronger signals from their immediate neighbors than from remote ones, it can determine whether it is closer to a DEV than other DEVs by comparing the relative signal strengths from them.

Then the DEV with the smallest standard deviation value calculated from all the strengths of the signals received from other DEVs is selected as the PNC. A DEV with the smallest standard deviation value is the very DEV which has the maximum number of close DEVs, meaning that it is the DEV in the center of the topology.

Then such relative signal strengths are exploited for the topological location of each DEV and are computed for a position value (PV) to be used for a PNC selection. The position value, as in (1), is computed by the mean and the standard deviation of a signal strength. A small standard deviation of a node means that the other devices are located evenly from the node. A small mean value of a node means that the other devices are located close to the node.

In Fig. 4, the standard deviation and the position value are listed in the table after the signal power of the DEVs in the topology is checked. The table shows that the standard deviation value of DEV4, which is located in the center of the topology, has the minimum value. It also shows that the standard deviation and the position values of the DEVs are different, depending on their locations in the topology. Once a DEV is selected as a PNC, which has the minimum position value, it transmits beacons with the minimum power which is enough to reach to the DEVs in the topology. By doing that, the PNC can save energy for transmission. 


\begin{tabular}{|c|c|c|c|c|c|c|c|c|c|c|}
\hline Srest & 1 & 2 & 3 & 4 & 5 & 6 & 7 & $\begin{array}{l}\text { Standard } \\
\text { Deviation }\end{array}$ & $\begin{array}{l}\text { Position } \\
\text { value }\end{array}$ \\
\hline 1 & & 6 & 6 & 6 & 2 & 4 & 1 & 1.8 & 2.31 \\
\hline 2 & 6 & & 5 & 6 & 3 & 6 & 4 & 1 & 5 \\
\hline 3 & 6 & 5 & & 6 & 5 & 4 & 3 & 0.9 & 5.37 \\
\hline 4 & 6 & 6 & 6 & & 5 & 6 & 5 & 0.43 & 13.17 \\
\hline 5 & 2 & 3 & 5 & 5 & & 4 & 6 & 1.1 & 3.78 \\
\hline 6 & 4 & 6 & 4 & 6 & 4 & & 6 & 1 & 5 \\
\hline 7 & 1 & 4 & 3 & 5 & 6 & 6 & & 1.8 & 2.31 \\
\hline
\end{tabular}

Fig. 4. Standard deviation value according to the topological location of a DEV

The position value (PV) is computed as following

$$
P V=\frac{m}{\sqrt{\frac{\left(x_{1}-m\right)^{2}+\left(x_{2}-m\right)^{2}+\cdots+\left(x_{n}-m\right)^{2}}{n}}}
$$

where $x_{i}$ represents the signal power of a DEV i and $m$ represents the average value of the signal powers of DEVs.

\subsection{PNC Mode Field for PNC Handover}

In IEEE 802.15.3 standard document, it is specified that transmitting power can be controlled when beacon, CAP and MCTA frames[1]. In this paper, we use such specification so that a node is chosen as a centered node in a topology and a solution is proposed for saving power of the chosen node. Specifically, there is Max TX Power Level field in the beacon frame, and an appropriate value is set in that field in order to control TX power of a PNC.

Each DEV controls transmission power for transmitting data to other DEVs. And also, transmitting power is determined by the link status between the two communicating nodes. At the beginning, each DEV transmits in full powering order to find the standard deviation value. The PNC mode field of the beacon frame in Fig. 5 is modified the one in Fig. 6. As shown in Fig. 6, SD(Standard Deviation) request filed is set to notify each DEV of using MAX TX Power level in order to measure the standard deviation value. After that, each DEV computes the standard deviation value based on the TX power that it listened to, and then sends the value to the PNC in order to let

\begin{tabular}{|c|c|c|c|c|c|}
\hline bits:b7-b5 & b4 & b3 & b2 & b1 & b0 \\
\hline Reserved & SEC mode & MCTA used & $\begin{array}{c}\text { CAP } \\
\text { association }\end{array}$ & $\begin{array}{c}\text { CAP } \\
\text { commands }\end{array}$ & CAP data \\
\hline
\end{tabular}

Fig. 5. Piconet mode field 


\begin{tabular}{|c|c|c|c|c|c|c|}
\hline bits:b7-b6 & b5 & b4 & b3 & b2 & b1 & bo \\
\hline Reserved & SD request & SEC mode & MCTA used & $\begin{array}{c}\text { CAP } \\
\text { association }\end{array}$ & $\begin{array}{c}\text { CAP } \\
\text { commands }\end{array}$ & CAP data \\
\hline
\end{tabular}

Fig. 6. Modified Piconet mode field

the PNC collects the values. The PNC, then uses the collected values when it needs to select a PNC when handover.

\section{Simulation}

The performance of the procedures proposed in this paper have been verified by the following simulation and their results. The assumption is that all of the DEVs in this simulation have limited and random battery remains. The simulation is performed for the handover algorithm in the IEEE 802.15.3 standard draft[1] and for the modified handover algorithm of ours. The DEVs in the simulation are assumed to be located randomly in a piconet. The parameters for the power consumption by a PNC for various operations used in the simulation are shown in Table 2. The PNC should use the full transmit power, while the other DEVs can save the transmit power, which is proportional to the communication distance to the destination from itself. In the simulation, the communications occur using beacons and the data packets with a fixed length. The power consumption is measured for the communications, not for the computations necessary for executing handover algorithm, because it is small enough to be negligible compared to that for communications.

Table 2. Power Consumption Parameters

\begin{tabular}{|c|c|}
\hline Transmit Power & $10 \mathrm{~mA}$ \\
\hline Receive Power & $7 \mathrm{~mA}$ \\
\hline Idle & $6.5 \mathrm{~mA}$ \\
\hline Sleep & $0.4 \mathrm{~mA}$ \\
\hline
\end{tabular}

Fig. 7 shows the duration of a piconet by the IEEE 802.15.3 standard and by our modified algorithm. The duration of a piconet is measured by the amount of time until there are only two DEVs have the energy remains, and all of the others are out of energy. The result shows that the performance of our modified algorithm improves about $20 \%$. The explanation for such results is that the power is less consumed because our modified algorithm is executed to find a PNC which is located as closely as possible to the center of a topology. It also shows that the duration improves as the number of DEVs in a piconet increases.

Fig. 8 shows the duration that all of the DEVs in a piconet have the power remains. It verifies that our modified algorithm performs better than the IEEE 802.15.3 handover algorithm. Fig. 9 and 10 show the amount of power consumption of each DEV in 
the simulation of Fig. 8 with the number of DEVs in a piconet being 10 and 20, respectively. The results present that using the modified handover algorithm consumes less energy than using the algorithm of the IEEE 802.15.3 standard. In addition, the results conform to the previous results in Fig. 8.

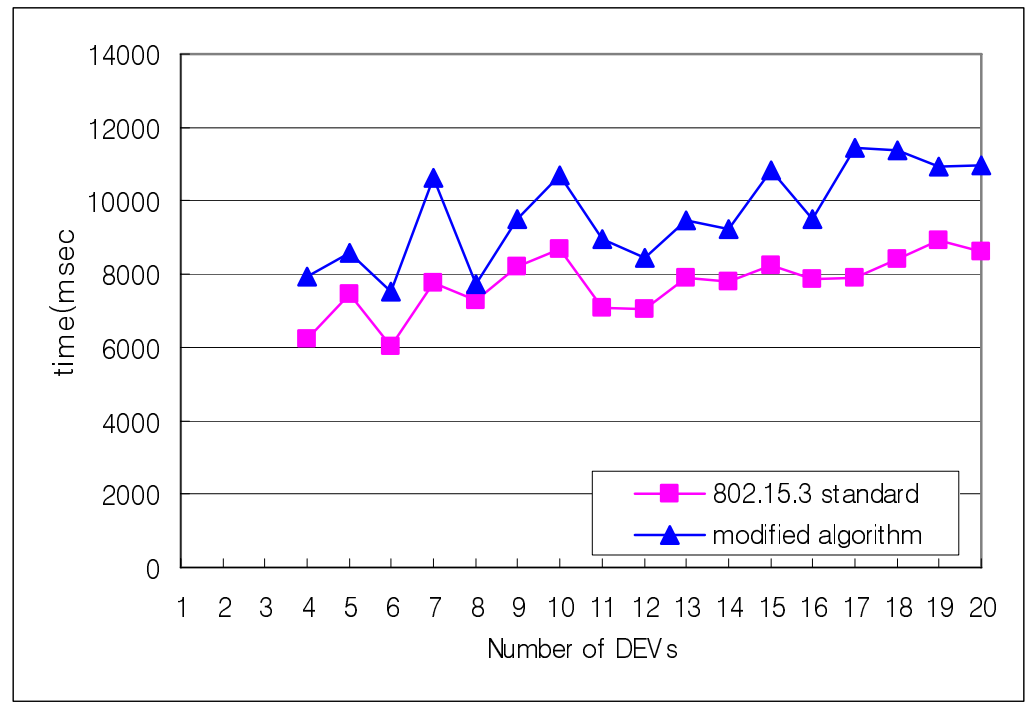

Fig. 7. Time duration until there are only two DEVs left with energy remains and the others are out of energy in a piconet

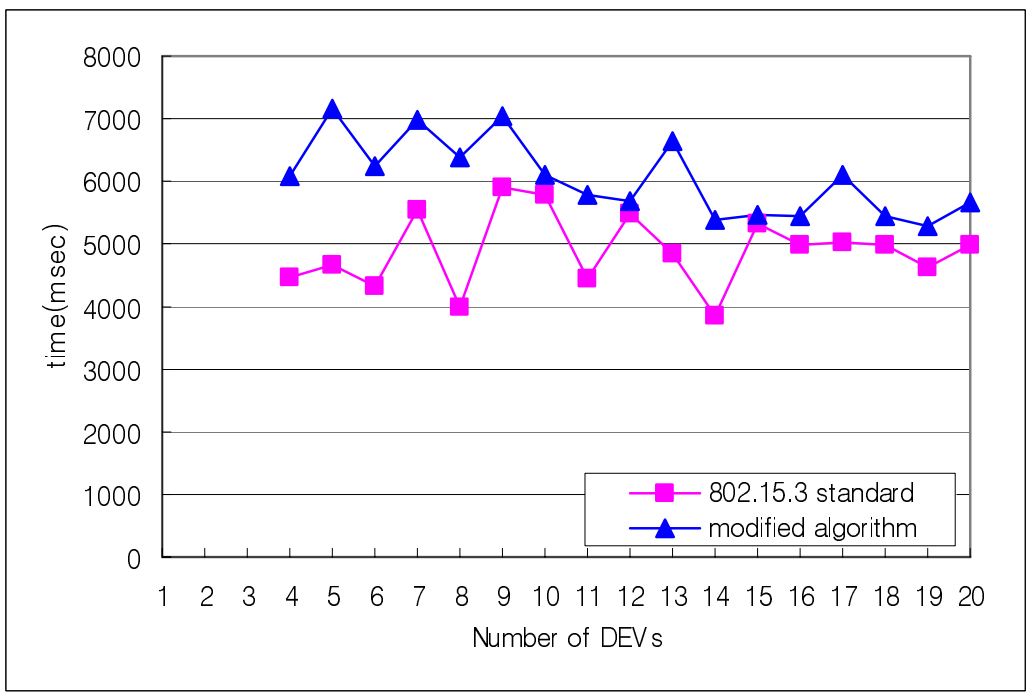

Fig. 8. Time duration until the first out-of-energy DEV occurs in a piconet 


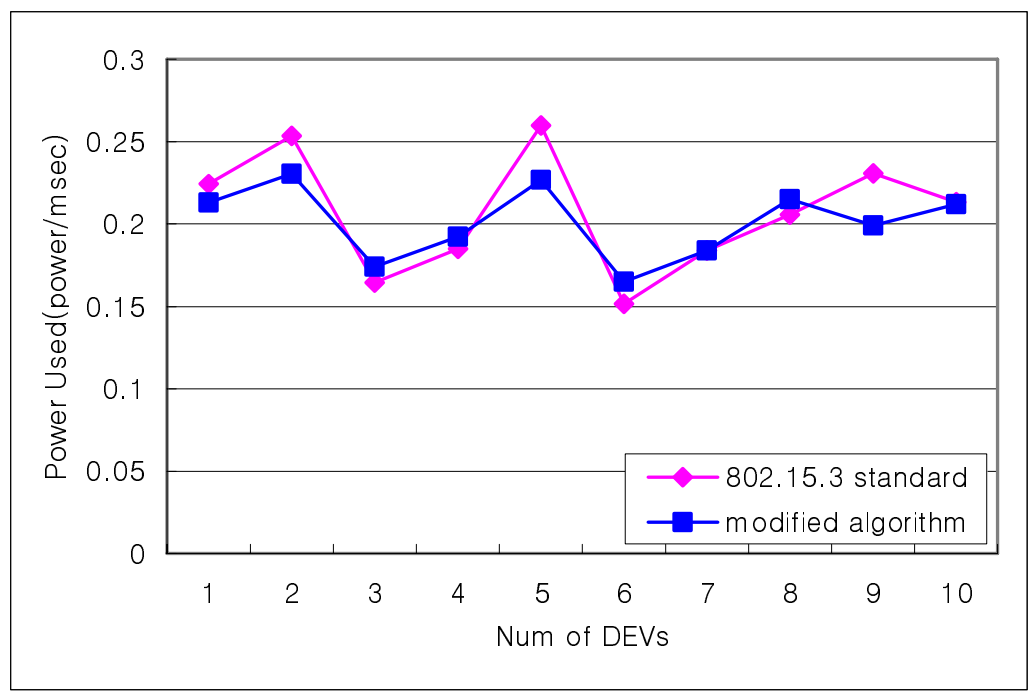

Fig. 9. The amount of energy consumption at each DEV when $10 \mathrm{DEV}$ s for the simulation of Fig. 8

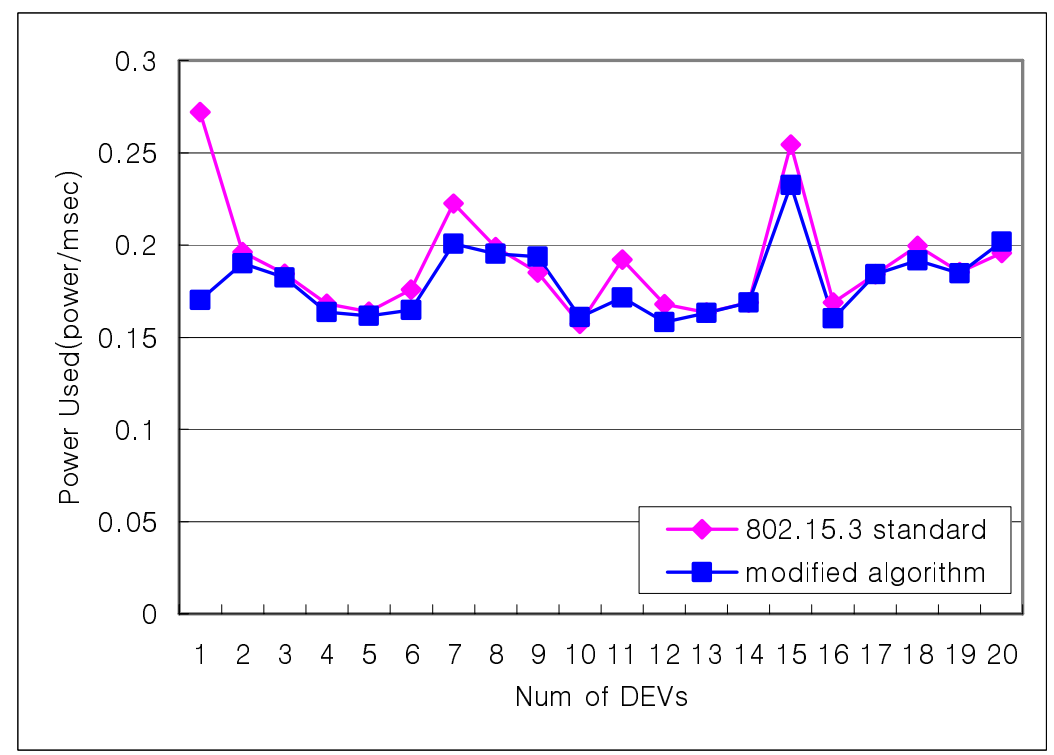

Fig. 10. The amount of energy consumption at each DEV when 20 DEV s for the simulation of Fig. 8 


\section{Closing Remarks}

In the IEEE 802.15.3 standard, there is a procedure for selecting a PNC for a piconet, where only the DEVs that have capabilities of performing PNC role are considered as a candidate for the PNC. Once a DEV is selected as a PNC, it is the device which consumes its power to perform the role of a PNC, so it might deplete its power faster than other DEVs. In this paper, we proposed a new algorithm for a PNC selection which takes energy efficiency into consideration. Our algorithm can be plugged in the IEEE 802.15.3 standard procedure as a component module, so the necessary modification is little, sustaining the compatibility with the standard procedure. The idea is to choose a DEV that is located as closely as possible to the topological center of a piconet. By doing that, the PNC consumes the energy for management communications as little as possible, so that the PNC can use its energy for its own communications. With our procedure, both the capability and the energy efficiency can be factors to select a PNC, compared to the fact that the capability is the only factor in the standard. To verify the performance improvements, we executed a comprehensive simulation and gave the results. As the results show, the amount of energy consumption of a PNC is reduced, and the duration of operation of a piconet lasts longer with our modified algorithm. In addition, the amount of the remaining energy is also greater than the standard algorithm. As a future work, we will apply the algorithm to the ad-hoc mobile networks to analyze its effectiveness.

\section{References}

1. "Draft Standard for Part 15.3: Wireless medium Access Control and Physical Layer Specifications for High rate Wireless Personal Area Networks (WPAN), “ Draft P802.15.3, Nov, 2001

2. Karaoguz, J., "High-rate wireless personal area networks", Communications Magazine, IEEE, Dec, 2001

3. Won Soo Kim and Il whan Kim, "A seamless coordinator switching(SCS) scheme for wireless personal area network(WPAN)" ,2003 IEEE International Conference, Aug, 2003

4. Harbin, S.A, and Rainer, B.E , "A low-power wireless mobile communications system," Vehicular Technology conference, June,1994

5. Jung-Won Kim, and Bambos, N, "Power control for multirate wireless networks with groupwise serial multiuser detection”, IEEE INFOCOM, Mar, 1998

6. Ingo Gruber, Oliver Knauf, Hui Li, "Performance of Ad Hoc Routing Protocols in Urban Environments", In Proceedings of European Wireless 2004, February, 2004

7. Stefano Basagni, "Mobile Ad hoc Networking", WILEY INTER SCIENCE, 2004

8. http://www.isi.edu/nsnam/ns 